\title{
Where to Encode: A Performance Analysis of x86 and Arm-based Amazon EC2 Instances
}

\author{
Roland Mathá*, Dragi Kimovski*, Anatoliy Zabrovskiy*, Christian Timmerer*†, Radu Prodan* \\ * Institute of Information Technology (ITEC), University of Klagenfurt, Austria \\ $\dagger$ Bitmovin, Klagenfurt, Austria
}

\begin{abstract}
Video streaming became an undivided part of the Internet. To efficiently utilise the limited network bandwidth it is essential to encode the video content. However, encoding is a computationally intensive task, involving high-performance resources provided by private infrastructures or public clouds. Public clouds, such as Amazon EC2, provide a large portfolio of services and instances optimized for specific purposes and budgets. The majority of Amazon's instances use $\times 86$ processors, such as Intel Xeon or AMD EPYC. However, following the recent trends in computer architecture, Amazon introduced Armbased instances that promise up to $40 \%$ better cost performance ratio than comparable $x 86$ instances for specific workloads. We evaluate in this paper the video encoding performance of $x 86$ and Arm instances of four instance families using the latest FFmpeg version and two video codecs. We examine the impact of the encoding parameters, such as different presets and bitrates, on the time and cost for encoding. Our experiments reveal that Arm instances show high time and cost saving potential of up to $33.63 \%$ for specific bitrates and presets, especially for the $\times 264$ codec. However, the $x 86$ instances are more general and achieve low encoding times, regardless of the codec.

Index Terms-Amazon EC2, Arm instances, AVC, Cloud computing, FFmpeg, Graviton2, HEVC, Performance analysis, Video encoding.

2021 IEEE. Personal use of this material is permitted. Permission from IEEE must be obtained for all other uses, in any current or future media, including reprinting/republishing this material for advertising or promotional purposes, creating new collective works, for resale or redistribution to servers or lists, or reuse of any copyrighted component of this work in other works.
\end{abstract}

\section{INTRODUCTION}

Multimedia streaming content [4], such as live and ondemand video and audio streams, is responsible for most Internet traffic today. Unfortunately, the Internet network connectivity can significantly change over time depending on many factors, such as client location, network congestion, or end-user device [27], [38]. The widely-used HTTP Adaptive Streaming (HAS) technology [30] encodes video content, divided into small segments, in multiple bitrate-resolution pairs to adapt to varying bandwidth fluctuations. Using HAS, a video segment reaches clients at different bitrate-resolution pairs for optimized quality of experience, depending on its network characteristics and technical capabilities (e.g., viewing device, video player) [10]. Additionally, a number of HAS implementations are codec-independent [30] and allow providers to choose from a set of codecs for video encoding, including Advanced Video Coding (AVC) [35], High-
Efficiency Video Coding (HEVC) [31], VP9 [28], AOMedia Video 1 (AV1) [12], and Versatile Video Coding (VVC) [9].

Creating segments of a single video encoded with different parameters (e.g., bitrate, resolution) for adaptive streaming is a computationally-intensive process that requires expensive high-performance computers or cheaper cloud resources rented on-demand [20], [34]. For example, Amazon EC2 provides different instance families optimized for specific purposes [24], such as the balanced general purpose $m$ instances, the compute-optimized $\mathrm{c}$ instances, the memory-optimized $r$ instances, and the burstable $t$ instances. To further optimize video encoding workloads at a convenient price, leading video encoding companies such as Bitmovin (https://bitmovin.com) combine on-demand instances with EC2 Spot instances for video encoding [1]. Nevertheless, choosing cloud instances for thousands of encoding tasks is critical and can strongly influence the costs for the video service providers [1], [19].

Recently, Amazon launched their second generation Graviton Arm-based processors. Graviton2 is a 64-core monolithic server chip that uses Arm's new Neoverse N1 cores, which is a derivation of the mobile Cortex-A76 cores. With the second generation of Graviton, Amazon EC2 promises higher performance at a lower cost compared to conventional $\times 86$ instances. According to Amazon, Graviton2 instances, such as $\mathrm{m} 6 \mathrm{~g}, \mathrm{c} 6 \mathrm{~g}, \mathrm{r} 6 \mathrm{~g}$ and $\mathrm{t} 4 \mathrm{~g}$, provide up to $40 \%$ better price performance over comparable instance types with Intel Xeon processor for video encoding tasks [3].

In this paper, we analyze the video encoding performance of EC2 instances based on the Graviton2 Arm and the $x 86$ processors using four instance families optimized for different purposes. As Graviton2 processors are relatively new and its software support is continuously improving, we conduct all experiments using FFmpeg [5] employing the following video codec implementations:

a) x264 codec: (H.264/AVC compression format) supported by the majority of end-user devices on the market [37].

b) $\times 265$ codec: (H.265/HEVC compression format) for further in-depth analysis with the newest FFmpeg version 4.3. The main contributions of our work are:

- We evaluate the relative video encoding time and cost differences between and $\times 86$ and Arm instance families;

- We identify the fastest and cheapest instance types among all instances of the same processor architecture.

- We provide a reference table indicating our recommendation for the fastest and cheapest encoding options for each 
instance family, preset, and bitrate.

The paper is organized into six sections. Section II discusses the related work. Section III presents the evaluation methodology. Section IV] describes the experimental design and evaluation scenarios, followed by the two codecs' experimental results in Section $\mathrm{V}-\mathrm{A}$ and Section $\mathrm{V}-\mathrm{B}$. Section $\mathrm{VI}$ summarizes our recommendations and discusses the results. Finally, Section VII concludes the paper and outlines the future work.

\section{RELATED WORK}

We review the state-of-the-art related to performance analysis and characterization of video encoding on cloud instances.

a) Cloud performance analysis: Li et al. [24] analyze the performance of heterogeneous cloud instances for encoding video streams and provide a model for quantifying the suitability of cloud instance types for various encoding tasks. Xiangbo et al. [23] present a performance analysis for improved scheduling of encoding tasks on cloud instances, which reduces the cost for the streaming service providers by $85 \%$. Timmerer et al. [33] present a performance analysis of the Bitmovin encoding platform, developed atop the MPEG-DASH open standard over multiple cloud instances. Based on this analysis, the encoding platform utilizes appropriate cloud instances to increase the average media throughput without stalling during operation.

b) Processor architecture: Jiang et al. [18] present a performance characterization of cloud instances based on the first generation of Graviton compared to a multitude of Intel Xeon processors. The work demonstrates that although the Graviton processor has the slowest encoding speed and scalability ratio, it provides cost savings of around $15 \%$ for the same encoding performance. Federman et al. [13] analyze the microarchitectural behavior of $\times 86$ processors for a set of video streaming workloads based on the $\times 264$ codec. They identify that video encoding suffers from a high amount of stalled instructions and cache misses, which leads to lower scalability and hindered execution. Magaki et al. [26] evaluate GPU and FPGA-based clouds' performance for video encoding with the ×265 codec and propose an application-specific integrated circuit tailored for this task.

c) Large scale video encoding: Jiang et al. [17] evaluate the performance of public cloud infrastructure for large-scale video encoding applications scaling up to one thousand virtual machines. Regarding video encoding benchmarks, Lottatini et al. [25] present a public suite tailored for cloud video services, which encompasses a set of representative videos and metrics that reflect user-perceived characteristics of the video streams, such as quality and encoding speed.

d) Gap analysis: The presented research works are segregated, utilize a single codec, and exclusively consider userspecific metrics, such a perceived video quality. In this work, we complement the related approaches by characterizing the performance of modern $\times 86$ and Arm architectures for a set of commonly used video codec implementations. Furthermore, we consider various video encoding presets (e.g., as known in $\times 264, \times 265)$ and provide a detailed analysis on the performance and the cost savings of using optimized instances in the cloud. Lastly, we examine the suitability of the Armbased processors for performing video encoding tasks.

\section{EVALUATION METHODOLOGY}

This section presents the performance evaluation methodology comprising two phases. 1) Encoding data generation describes the selection of representative video sequences, identifies video codecs, and selects encoding parameters. 2) Instance selection and metric definition describes the selection of cloud instances based on the processor micro-architecture and defines the relevant performance evaluation metrics.

\section{A. Encoding data generation}

Encoding data generation involves video sequence, video codec, and encoding parameter selection.

1) Video sequence selection: We select video segments with a duration below $10 \mathrm{~s}$ according to HAS requirements and industry best practices [22]. The segment length is a key parameter in HAS, as each video segment starts with a random access point to enable adaptive and dynamic switching to other representations (bitrate - resolution pairs) at segment boundaries [11]. In addition, the selected video segment must contain movements that exploit different features of the video coding algorithms. Therefore, we use spatial and temporal information metrics to select a video segment and identify the computation requirements for its encoding [15].

a) Spatial Information (SI): measures the spatial complexity of video frames through the physical position of an object in the frame and its spatial relationship to other objects:

$$
S I=\max _{\forall F_{n}}\left\{\sigma\left[\operatorname{Sobel}\left(F_{n}\right)\right]\right\},
$$

where $F_{n}$ is the luminance component of the video frame at time instance $n, \sigma$ is the standard deviation across all the pixels in the Sobel filter, and the max operator calculates the maximum standard deviation of all frames in a video sequence. A high SI value indicates complex spatial relations between multiple objects and higher differences between subsequent frames, which increases the complexity of the encoding tasks and leads to longer encoding times.

b) Temporal Information (TI): shows the amount of motion in a video content, calculated as the maximum standard deviation $\sigma$ of a motion difference function $M_{n}(i, j)$. This function represents the difference in luminance for two sequential frames $F_{n}$ and $F_{n-1}$ across all the pixels $(i, j)$ :

$$
\begin{gathered}
T I=\max \left\{\sigma\left[M_{n}(i, j)\right]\right\} ; \\
M_{n}(i, j)=F_{n}(i, j)-F_{n-1}(i, j),
\end{gathered}
$$

where $F_{n}(i, j)$ is the luminance of the frame pixel $(i, j)$ at time instance $n$ in the video sequence. A high TI value indicates higher motion differences between the video segment frames, which requires more computational resources for performing the encoding tasks. 
Table I: Bitrate ladder (bitrate - resolution pairs).

\begin{tabular}{|c|c|c||c|c|c|}
\hline$\#$ & Bitrate [kbps] & Resolution & $\#$ & Bitrate [kbps] & Resolution \\
\hline 1 & 100 & $256 \times 144$ & 11 & 4300 & $1920 \times 1080$ \\
2 & 200 & $320 \times 180$ & 12 & 5800 & $1920 \times 1080$ \\
3 & 240 & $384 \times 216$ & 13 & 6500 & $2560 \times 1440$ \\
4 & 375 & $384 \times 216$ & 14 & 7000 & $2560 \times 1440$ \\
5 & 550 & $512 \times 288$ & 15 & 7500 & $2560 \times 1440$ \\
6 & 750 & $640 \times 360$ & 16 & 8000 & $3840 \times 2160$ \\
7 & 1000 & $768 \times 432$ & 17 & 12000 & $3840 \times 2160$ \\
8 & 1500 & $1024 \times 576$ & 18 & 17000 & $3840 \times 2160$ \\
9 & 2300 & $1280 \times 720$ & 19 & 20000 & $3840 \times 2160$ \\
10 & 3000 & $1280 \times 720$ & & & \\
\hline
\end{tabular}

2) Video codec selection: We identify $\times 264$ and $\times 265$ [37] as the most widely spread codecs for executing video encoding tasks, deployed by more than $90 \%$ of the video streaming industry [16]. The $\times 265$ video codec typically requires more computing resources than $\times 264$ but achieves a higher video quality for the same encoding parameters [14].

3) Encoding parameters selection: We select 19 bitrates from $100 \mathrm{kbps}$ to $20 \mathrm{Mbps}$ (see Table $\Phi$ ) and nine encoding presets that define the quality to encoding speed ratio: ultrafast, superfast, veryfast, faster, fast, medium (default preset), slow, slower, and veryslow [6]. A slower preset uses more features for the same bitrate, which leads to a relatively slower encoding speed and better video quality [29]. Similarly, faster presets produce lower video quality. According to the official FFmpeg video encoding guide, we do not use the placebo preset that does not provide a significant quality improvement compared to the veryslow preset according to the official FFmpeg video encoding guide [6].

\section{B. Instance selection and metric definition}

1) Instance type selection: We selected instance types based on three commonly used processors for video encoding.

a) Intel Xeon Platinum: is a multi-purpose processor based on the latest extension of the $\times 86$ architecture with the Advanced Vector Extension (AVX-512) instruction set. It is a 28-core server chip that can execute 56 concurrent threads.

b) $A M D E P Y C$ : is a multi-purpose processor based on the $\times 86$ Zen architecture. For the majority of instance types, AWS provides the first generation EPYC processor with up to 32 cores and 64 concurrent threads. However, for the $\mathrm{C}$ instances, AWS provides the second-generation EPYC processors with up to 64 cores and 128 threads per server chip.

The $x 86$ processors of Intel and AMD represent $87 \%$ of the cloud instances [2] and the majority of personal computers.

c) Graviton2: is the second generation of Graviton Arm processors recently released by Amazon. It is a 64-core monolithic server chip that uses Arm's new Neoverse N1 cores, derived from the mobile Cortex-A76 cores. The Arm processors dominate the mobile segment with a $90 \%$ market share [2]. Nevertheless, there is a trend [32] from leading companies, such as Apple and Amazon, for using Arm-based processors to power personal computers and cloud instances.

d) Instance families: Based on these processor architectures, we select four instance families from the Amazon EC2 cloud (see Table IIT) [24]: 1) balanced general purpose $m$ instances, 2) compute-optimized $\mathrm{C}$ instances, memory-optimized $r$ instances, and 3) burstable $t$ instances. We selected eight vCPUs for all instance types, corresponding to the largest available sizes of $t 3, t 3 a$, and $t 4 g$ instances. For a fair comparison, we equalize the number of vCPUs. However, the memory size of different instance types might still differ due to their instance type definitions. Nonetheless, the carefully selected video segment's encoding does not exceed the smallest memory size in our set of instances.

2) Evaluation metrics: We compare the new Arm instances with the Intel and AMD based $\times 86$ instances using three metrics.

a) Relative encoding time: $\Delta t_{e n c}\left(V_{b, p}\right)$ of a video segment $V_{b, p}$ with a bitrate $b$ and a preset $p$ is the normalized time difference of the Arm encoding time $t_{A r m}\left(V_{b, p}\right)$ to the reference $\times 86$ encoding time $t_{x 86}\left(V_{b, p}\right)$ :

$$
\Delta t_{\text {enc }}\left(V_{b, p}\right)=\frac{t_{A r m}\left(V_{b, p}\right)-t_{x 86}\left(V_{b, p}\right)}{t_{x 86}\left(V_{b, p}\right)} \cdot 100 .
$$

A positive relative encoding time indicates that the Arm instance is slower than the reference $\times 86$ instance, while a negative value indicates that $\mathrm{Arm}$ is faster.

b) Encoding cost: $c_{e n c}\left(V_{b, q}\right)$ of a video segment $V_{b, q}$ is the product between the instance price $c_{i}$ (in $\$$ ) per second and the segment encoding time $t\left(V_{b, q}\right)$ in seconds:

$$
c_{\text {enc }}\left(V_{b, q}\right)=c_{i} \cdot t\left(V_{b, q}\right),
$$

Although cloud providers typically charge for their on-demand instances on an hourly basis, we scale the price down to seconds for a more fine-grained encoding cost understanding.

c) Relative encoding cost: $\Delta c_{e n c}\left(V_{b, p}\right)$ for a video segment $V_{b, p}$ with a bitrate $b$ and a preset $p$ is the normalized difference of the Arm encoding cost $c_{A r m}\left(V_{b, p}\right)$ to the reference $\times 86$ encoding cost $c_{x 86}\left(V_{b, p}\right)$ :

$$
\Delta c_{e n c}\left(V_{b, p}\right)=\frac{c_{A r m}\left(V_{b, p}\right)-c_{x 86}\left(V_{b, p}\right)}{c_{x 86}\left(V_{b, p}\right)} \cdot 100 .
$$

Similarly to relative encoding time, a positive relative encoding cost indicates that Arm is more costly than the reference $\times 86$ instance, while a negative value indicates that $\mathrm{Arm}$ is cheaper.

\section{EXPERIMENTAL DESIGN}

This section describes the implementation and evaluation scenarios of the encoding methodology.

\section{A. Video sequence selection}

We selected for encoding a two-second segment from a computer-animated movie [36] with high TI and SI metrics [21], as described in Section [II] The selected video segment's TI metric has a value of 22 , which is three times higher than the average TI value (8.2) of the other movie segments. This implies high motion between the frames. In turn, the SI metric of this segment has a value of 18.1, which is slightly above the average SI value (16.3) of all movie segments, which implies higher spatial complexity. 
Table II: Experimental Amazon EC2 Cloud instance types.

\begin{tabular}{|c|c|c|c|c|c|c|c|c|}
\hline Instance type & Architecture & ${ }_{v C P U s}$ & Memory [GiB] & Storage [GiB] & Network & Physical processor & Clock $[\mathrm{GHz}]$ & Price $[\$ / \mathrm{h}]$ \\
\hline m5.2xlarge & \multirow{4}{*}{ 64-bit x86 } & \multirow{4}{*}{8} & 32 & \multirow{4}{*}{ EBS } & \multirow{4}{*}{$\begin{array}{l}\leq 10 \mathrm{Gbps} \\
\leq 5 \mathrm{Gbps}\end{array}$} & 1st or 2 nd generation & $\leq 3.1$ & 0.383 \\
\hline c5.2xlarge & & & 32 & & & Intel Xeon Platinum 8000 & $\leq 3.4$ & 0.34 \\
\hline r5.2xlarge & & & 64 & & & series (Skylake-SP or & $\leq 3.1$ & 0.504 \\
\hline t $3.2 \times$ large & & & 32 & & & Cascade Lake) & $\leq 3.1$ & 0.3341 \\
\hline m5a.2xlarge & \multirow{4}{*}{ 64-bit x86 } & \multirow{4}{*}{8} & 32 & \multirow{4}{*}{ EBS } & \multirow{4}{*}{$\begin{array}{l}\leq 10 \mathrm{Gbps} \\
\leq 5 \mathrm{Gbps}\end{array}$} & AMD EPYC 7000 series & $\leq 2.5$ & 0.344 \\
\hline c $5 a .2 x l a r g e$ & & & 16 & & & AMD EPYC 7002 (2nd gen.) & $\leq 3.3$ & 0.308 \\
\hline r5a.2xlarge & & & 64 & & & AMD EPYC 7000 series & $\leq 2.5$ & 0.452 \\
\hline t3a.2xlarge & & & 32 & & & AMD EPYC & $\leq 2.5$ & 0.3008 \\
\hline $\mathrm{m} 6 \mathrm{~g} .2 \mathrm{xlarge}$ & \multirow{4}{*}{ 64-bit Arm } & \multirow{4}{*}{8} & 32 & \multirow{4}{*}{ EBS } & \multirow{4}{*}{$\begin{array}{l}\leq 10 \mathrm{Gbps} \\
\leq 5 \mathrm{Gbps}\end{array}$} & \multirow{4}{*}{ AWS Graviton2 } & \multirow{4}{*}{$\leq 2.5$} & 0.308 \\
\hline c6g.2xlarge & & & 16 & & & & & 0.272 \\
\hline r6g.2xlarge & & & 64 & & & & & 0.4032 \\
\hline t4g.2xlarge & & & 32 & & & & & 0.2688 \\
\hline
\end{tabular}

\section{B. Encoding software}

We perform the encoding for $\mathrm{AVC}$ and $\mathrm{HEVC}$ video codecs using the libx264 (short $\times 264$ ) and libx265 (short x265) FFmpeg [5] library, as follows:

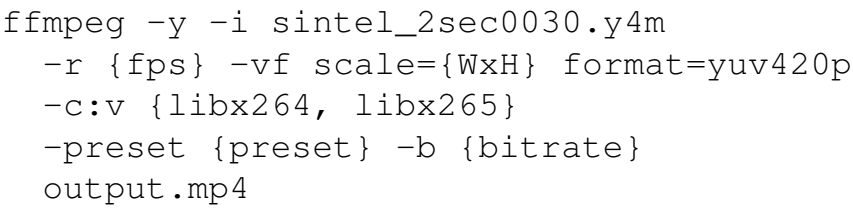

a) fps: is the number of video frames per second, which is the same as in the original video segment (i.e., 24);

b) $W \times H$ : specifies the width $\mathrm{W}$ and height $\mathrm{H}$ (in pixels) of the encoded video segment (see Table I);

c) preset: defines the encoding preset (e.g., fast, slow);

d) bitrate: in a encoded video file measured in kbps, as defined in Table I]

We used the latest FFmpeg version 4.3 which uses the libx265 version 3.4 with Huawei enhancements for faster Arm encoding [8]. We carefully select the number of encoding threads equal to the number of available vCPUs of each instance type. We run all instances in the US East Amazon EC2 region with two default Ubuntu Server 18.04 LTS images that include Python packages: ami-0ac80 df 6 eff 0 e $70 b 5$ for 64-bit $\times 86$ instances, and ami-0d221091ef7082bcf for 64-bit Arm instances. We manually deployed the latest FFmpeg version 4.3 to replace the default 3.4 .6 version of Ubuntu 18.04 LTS.

\section{Evaluation scenarios}

We created two evaluation scenarios for each of the codec. We repeated all experiments five times for a total of 10,260 experiments for both $\times 264$ and $\times 265$ codec. This translates into a total cloud instance time of over $260 \mathrm{~h}$. We report the average values of the metrics described in the following paragraphs.

1) Instance family: comparison uses two metrics:

a) Relative encoding time: difference $\Delta t_{e n c}\left(V_{b, p}\right)$ of each $\times 86$ and Arm instance from the same instance family, as presented in Table II (i.e., m, c, r, t);

b) Relative encoding cost: difference $\Delta c_{e n c}\left(V_{b, p}\right)$ across both processor architectures from the same instance family.

2) Processor architecture: comparison uses two metrics: a) Fastest encoding time: of Arm $t_{A r m}\left(V_{b, p}\right)$ and $\times 86$ instances $t_{x 86}\left(V_{b, p}\right)$ independent of the instance family.

b) Lowest encoding cost: $c_{e n c}\left(V_{b, p}\right)$ across all instance families from the same processor architecture.

\section{Evaluation RESULts}

We present the encoding results that compare the $\times 86$ and Arm instances for the $\times 264$ and $\times 265$ codecs, following the evaluation scenarios from Section IV-C. We use heatmaps to simplify the three-dimensional visualization of the encoding time and cost dependency on the encoding bitrates and presets.

\section{A. $x 264$ Codec}

\section{1) Instance family:}

a) Relative encoding time: Figure 1a displays the relative encoding time $\Delta t_{\text {enc }}\left(V_{b, p}\right)$ between Arm and $\times 86$ instances (Intel and AMD) with various encoding presets and bitrates. We observe that the $\times 86 \mathrm{C}$ instances achieve for all presets and bitrates on average $30,78 \%$ faster encoding times than the Arm $\mathrm{c} 6 \mathrm{~g}$ instances. For the other instance families, the $\times 86$ instances are faster than the Arm instances, primary for lower bitrates $(\leq 8 \mathrm{Mbps})$ and presets between "ultrafast" and "veryfast". The Arm instances $t 4 \mathrm{~g}, \mathrm{~m} 6 \mathrm{~g}$ and $r 6 \mathrm{~g}$ reveal faster encoding times than the Intel $\times 86$ instances, especially for presets between "very fast" and "fast", and for bitrates higher than $750 \mathrm{kbps}$. In particular, $\mathrm{m} 6 \mathrm{~g}$ and $\mathrm{r} 6 \mathrm{~g}$ achieve up to $7.51 \%$, while $t 4 \mathrm{~g}$ achieves up to $17.82 \%$ faster encoding times than the Intel $\times 86$ instances. Considering the $\times 86$ instances of AMD, the Arm instances $t 4 \mathrm{~g}, \mathrm{~m} 6 \mathrm{~g}$ and $\mathrm{r} 6 \mathrm{~g}$ achieve faster encoding times especially for presets between "very fast" and "very slow", and for bitrates higher than 750 kbps.

Overall, t $4 \mathrm{~g} .2 \mathrm{xlarge}$ shows the shortest relative encoding time due to the burstable behavior.

b) Relative encoding cost: Beside relative encoding times, we closely analyze the relative encoding costs $\Delta c_{e n c}\left(V_{b, p}\right)$ with various encoding presets and bitrates (see Figure $1 \mathrm{~b}$ ). For $\mathrm{m}, \mathrm{r}$, and $t$ instances, the Arm instances reveal the highest cost saving potential for presets between "very fast" and "fast", and bitrates over $750 \mathrm{kbps}$. For example, t $4 \mathrm{~g} .2 \times$ large shows on average $19.67 \%$, r $6 \mathrm{~g} .2 \times$ large $13.75 \%$, and $\mathrm{m} 6 \mathrm{~g} .2 \mathrm{xlarge} 12.84 \%$ lower encoding cost 

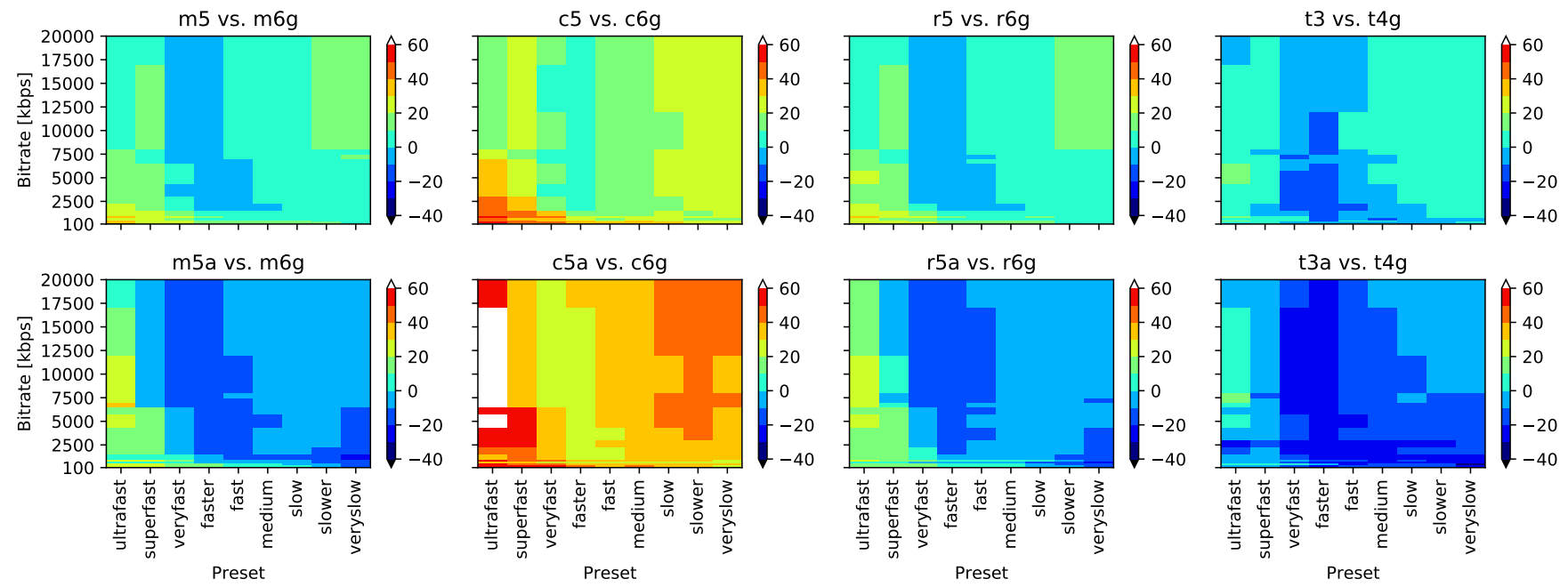

(a) Encoding times.
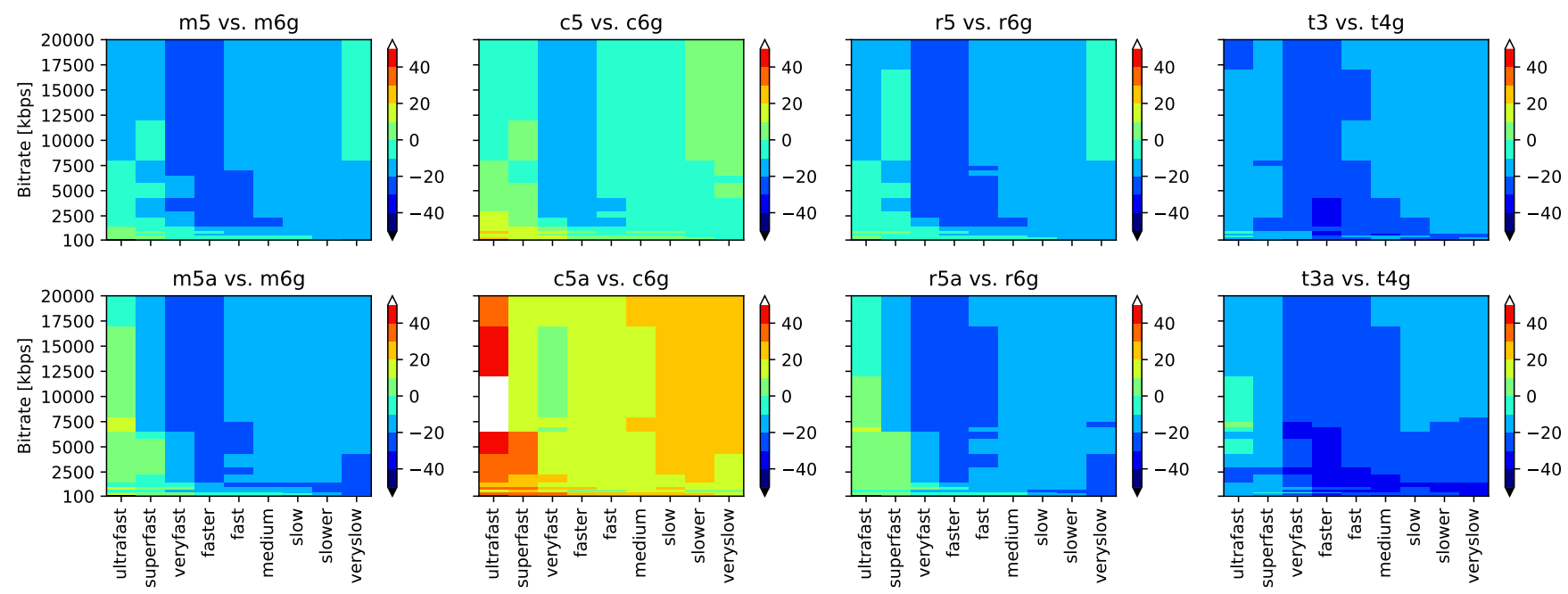

(b) Encoding costs.

Figure 1: Relative encoding time and cost for four instance families with different presets, bitrates, and x264 codec.

than the corresponding $\times 86$ instances. The $c$ instances, especially the AMD based $\mathrm{c} 5 \mathrm{a}$, show highest cost saving potential for all presets and bitrates. The combination of lower cost and faster encoding performance than Intel based $c 5$ make the c 5 a to the first choice for the $\times 264$ codec.

c) Recommendation: For fast encoding, we recommend using $\times 86$ instances for bitrates lower than $8 \mathrm{Mbps}$ and presets between "ultrafast" and "veryfast". Arm instances are faster for bitrates higher than $750 \mathrm{kbps}$ and presets between "very fast" and "fast". For a low encoding cost, we recommend Arm over $\mathrm{x} 86$ for $\mathrm{m}, \mathrm{r}$, and $\mathrm{t}$ instances. However, for $\mathrm{c}$ instances, we recommend $\times 86$, and especially the AMD based $c 5 \mathrm{a}$, over Arm.

2) Processor architecture:

a) $\times 86$ encoding time: The $\mathrm{c}$ instance family achieves the fastest encoding times in all experiments. Considering only Intel's $\times 86$, c5 is on average $10.44 \%$ faster than other three
Intel $\times 86$ instances due to its higher clock speed. However, the direct comparison of the Intel c5 and the AMD c 5 a reveal, that the AMD based c instance is on average $10.17 \%$ faster. This performance difference is expected because the computeoptimized $\mathrm{C} 5 \mathrm{a}$ instances use the second generation AMD EPYC processors, which are based on newer architecture and production processes than the Intel Xeon processors used in c5.

b) Arm encoding time: Figure 2a shows the fastest encoding Arm instances. Overall, the encoding times among Arm instances are negligibly small with encoding time differences of less than $0.85 \%$ on average. Furthermore, Figure $2 \mathrm{a}$ confirms that $\mathrm{m} 6 \mathrm{~g} .2 \mathrm{x}$ large is fastest in $90 \%$ of the experiments with $1.15 \%$ faster average encoding times. The $t 4 \mathrm{~g} .2 \mathrm{xlarge}$ instance is fastest for bitrates higher than $12 \mathrm{Mbps}$ and presets between "slower" and "veryslow". The 


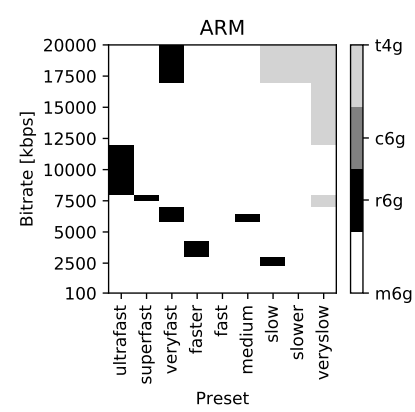

(a) Fastest.

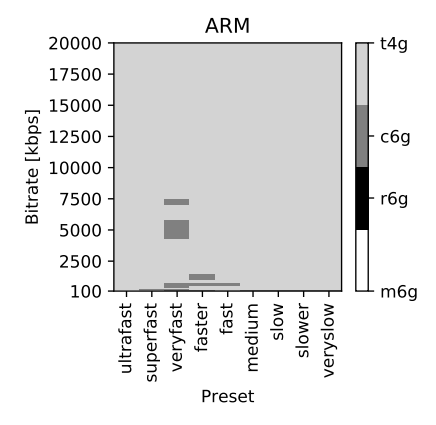

(b) Cheapest.
Figure 2: Fastest and cheapest encoding architecture per instance type for different presets, bitrates, and $\times 264$ codec.

r $6 \mathrm{~g} .2 \mathrm{x}$ large instances are fastest in few cases with no detectable pattern, mainly for bitrates between $2.5 \mathrm{Mbps}$ and $12 \mathrm{Mbps}$, and presets between "ultrafast" and "slow".

c) Encoding cost: The $\mathrm{c}$ instance and especially the AMD-based $c 5 a$ instance achieves the lowest $\times 86$ encoding costs in all experiments thanks to its fast encoding times and low price compared to the other three $\times 86$ instances. Figure $2 \mathrm{~b}$ shows the lowest encoding cost for Arm instances. In particular, the $t 4 \mathrm{~g} .2 \times$ large instance achieves the lowest encoding costs, followed by $\mathrm{c} 6 \mathrm{~g} .2 \mathrm{x}$ large. The low encoding cost of t $4 \mathrm{~g} .2 \mathrm{xlarge}$ in the majority of experiments is due to its low price and burstable performance.

d) Recommendation: Among $\times 86$ instances, we recommend using $\mathrm{c}$ instances for fastest encoding and lowest cost. Among $c$ instances, we recommend to prioritize c $5 \mathrm{a}$ over c5 instances, as c $5 \mathrm{a}$ are based on latest processor architecture from AMD. Among Arm instances, we recommend $\mathrm{m} 6 \mathrm{~g} .2 \times \mathrm{large}$ for fastest encoding times, and t $4 \mathrm{~g} .2 \times$ large for lowest encoding cost.

\section{B. $x 265$ codec}

To analyze the different codecs' impact, we repeat the same set of experiments using the $\times 265$ codec.

1) Instance family:

a) Relative encoding time: Figure 3 reveals that the Arm instances are on average $329.42 \%$ slower than the $\times 86$ instances in all experiments. We observe higher relative encoding times of at least $279.92 \%$ for presets between "slow" and "veryslow" and lower for presets between "ultrafast" and "superfast". In particular, t $4 \mathrm{~g} .2 \mathrm{xlarge}$ shows the smallest difference of $16.48 \%$ for a bitrate of $100 \mathrm{kbps}$, and c5a the highest differences of up to $847.67 \%$ compared to Arm $\mathrm{c} 6 \mathrm{~g}$ instance for presets between "slow" and "veryslow". The m6g.2xlarge and $r 6 \mathrm{~g} .2 \times$ large instances achieve comparable results depending on the $\times 86$ processor. We identify a higher average relative encoding time difference of $360.79 \%$ for Intel based instances, and $229.76 \%$ for AMD based instances.

In contrast to the $\times 264$ codec, Arm instances show in all experiments slower relative encoding times. Moreover, we identify different areas in the heatmaps with small relative encoding time for both codecs. For example, the x 265 codec shows the smallest difference for areas with low bitrate and "ultrafast" preset, where the $\times 264$ codec shows highest $\Delta t_{\text {enc }}\left(V_{b, q}\right)$.

b) Relative encoding cost: For completeness, Figure $3 \mathrm{~b}$ depicts the relative encoding cost $\Delta c_{e n c}\left(V_{b, p}\right)$ between $\times 86$ and Arm instances with various encoding presets and bitrates. The Arm instances introduce $382.58 \%$ higher encoding costs on average than the corresponding $\times 86$ instances in all experiments.

As Arm instances imply $31.66 \%$ to $635.01 \%$ higher encoding costs compared to Intel-based and $4.09 \%$ to $736.90 \%$ for AMD basedx 86 instances. Therefore, we do not identify any cost-saving potential. We explain the higher costs due to the high relative encoding time, as presented in the previous section.

c) Recommendation: The experimental results confirm that the $\times 265$ support in FFmpeg 4.3 is not yet optimized for Arm instances. We recommend using $\times 86$ instances.

2) Processor architecture:

a) $\times 86$ encoding time: Figure $4 \mathrm{a}$ shows that the $\mathrm{c}$ family delivers the fastest encoding time in all experiments on $\times 86$ instances. Especially, the $\mathrm{C} 5 \mathrm{a}$ instance with AMD processors is on average $15.65 \%$ faster than all other $\times 86$. In particular, c $5 \mathrm{a}$ is on average $31.64 \%$ faster than $t$ instances, $27.19 \%$ faster than $\times 86 \mathrm{~m}$ and $\mathrm{r}$ instances, and $4.67 \%$ faster than $\mathrm{c} 5$ instances with Intel processor.

b) Arm encoding time: Figure $4 \mathrm{~b}$ shows that $\mathrm{m} 6 \mathrm{~g} .2 \mathrm{xlarge}$ is fastest in $66.08 \%$ of the experiments, followed by $t 4 \mathrm{~g} .2 \times \mathrm{large}$ in $23.39 \%$. Overall, the relative encoding time among the Arm instances is negligibly small and in the range of measurement noise. More precisely, $\mathrm{m} 6 \mathrm{~g} .2 \times$ large is on average only $0.21 \%$, t $4 \mathrm{~g} .2 \times 1$ arge $0.13 \%, r 6 \mathrm{~g} .2 \mathrm{x}$ large $0.23 \%$, and $\mathrm{c} 6 \mathrm{~g} .2 \mathrm{xlarge} 0.07 \%$ faster.

c) Encoding cost: For all experiments, c5a.2xlarge achieves lowest encoding costs among $\times 86$ instances, and t $4 \mathrm{~g} .2 \mathrm{xlarge}$ among Arm instances. Specifically, the c5a instance with AMD processors reveals on average $15.65 \%$ lower cost than all other $\times 86$ instances and $13.65 \%$ lower average cost than the corresponding $c 5$ instance with Intel processors. For comparison, the $4 \mathrm{~g} .2 \mathrm{x}$ large reports only $1.34 \%$ lower cost among all Arm instances.

d) Recommendation: The c5a.2xlarge instance shows the fastest encoding times and the lowest encoding cost for $\times 86$ instances in all experiments. Among the Arm instances, $\mathrm{t} 4 \mathrm{~g} .2 \mathrm{xlarge}$ competes with $\mathrm{m} 6 \mathrm{~g} .2 \times \mathrm{large}$ for the fastest encoding time. However, $\mathrm{t} 4 \mathrm{~g} .2 \mathrm{xl}$ large achieves the lowest encoding cost in all experiments.

\section{SUMmARY AND Discussion}

\section{A. Summary}

We summarize in this section our recommendations related to the video encoding time and cost for both codecs and processor architectures. In Figure 5 we provide accurate visual 

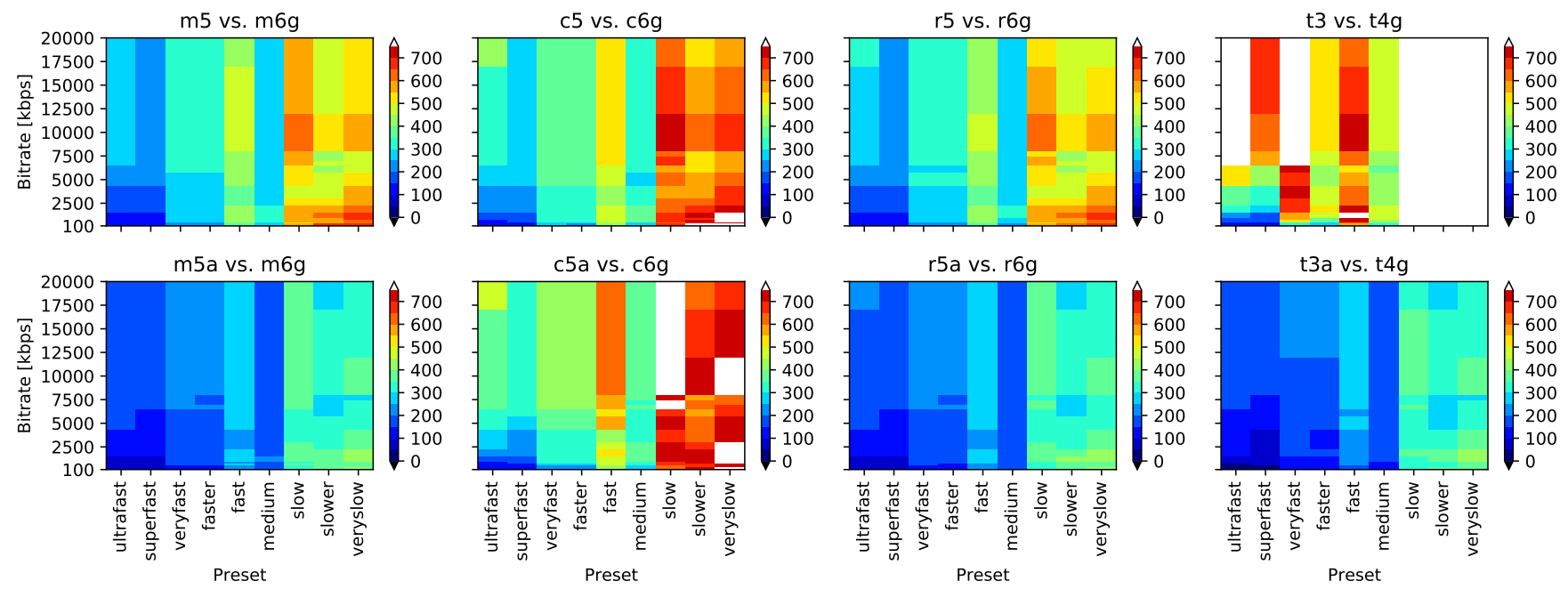

(a) Encoding times.
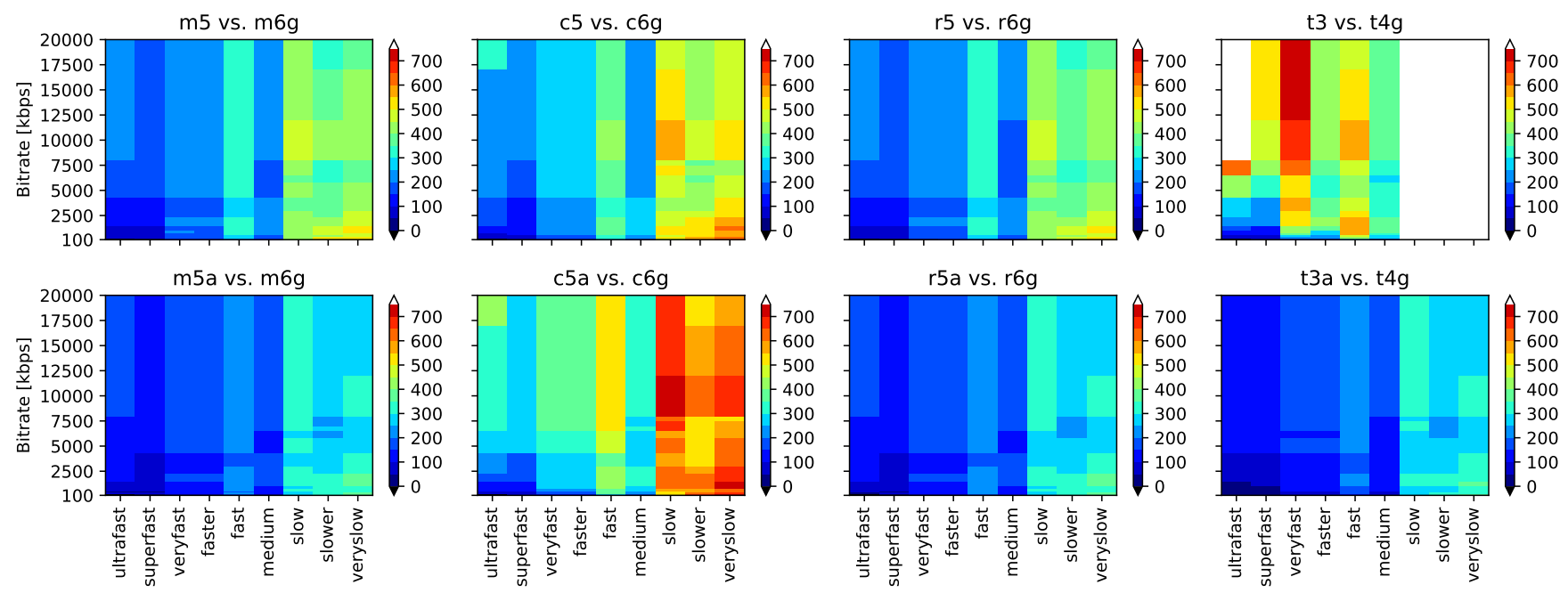

(b) Encoding costs.

Figure 3: Relative encoding time and cost for four instance families with different presets, bitrates, and x265 codec.

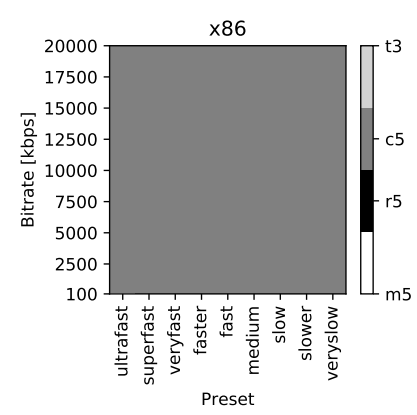

(a) $\times 86$ instances.

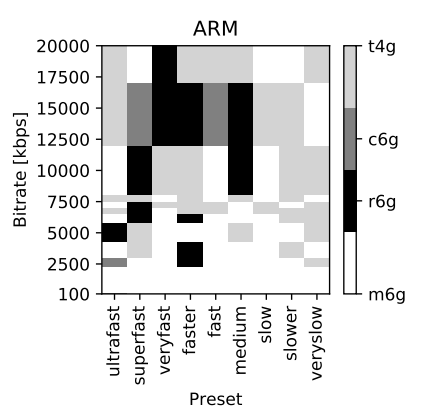

(b) Arm instances.

Figure 4: Fastest encoding instance for different encoding presets, bitrates, and $\times 265$ codec.

reference tables indicating the fastest and cheapest options for all evaluated instance families, presets, and bitrates for the $\times 264$ codec. As the results for $\times 265$ codec report a clear best performing instance family for the fastest time and lowest cost, independently from the preset and bitrate, we describe these results only textually.

\section{1) Fast encoding:}

a) $\times 264$ codec: Overall, we recommend $\times 86$ instances especially for bitrates lower than $8 \mathrm{Mbps}$ and presets between "ultrafast" and "veryfast". As depicted in Figure 5a, we recommend Arm instances mainly for bitrates higher than $750 \mathrm{kbps}$ and presets between "very fast" and "fast". Among all $\times 86$ instances, we recommend to use $c$ instances and especially to prioritize the AMD based $c 5$ a over the Intel based c5 instance. Among all Arm instances,the $\mathrm{m} 6 \mathrm{~g} .2 \times 1$ arge instance is the fastest, but with an advantage of only $1.15 \%$ on average. 

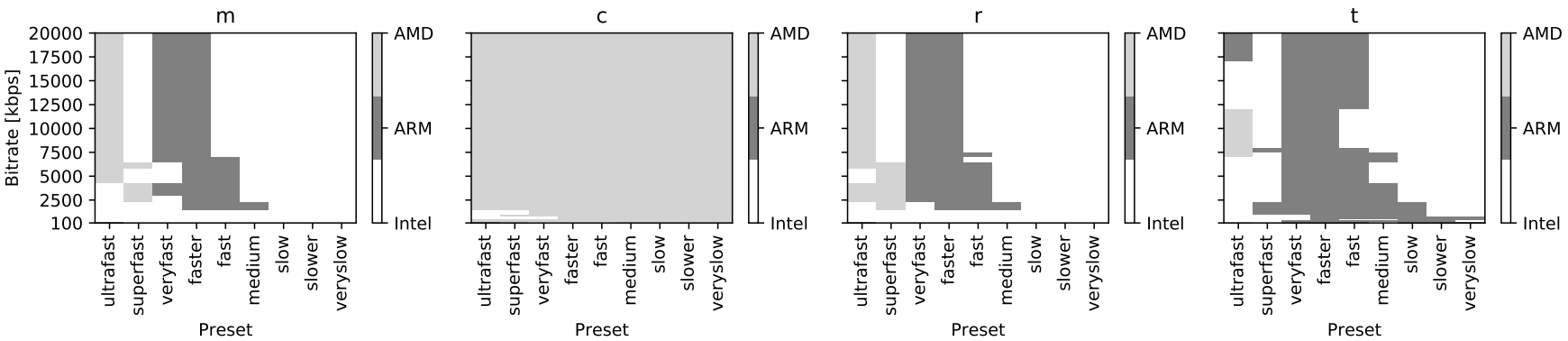

(a) Fastest encoding.
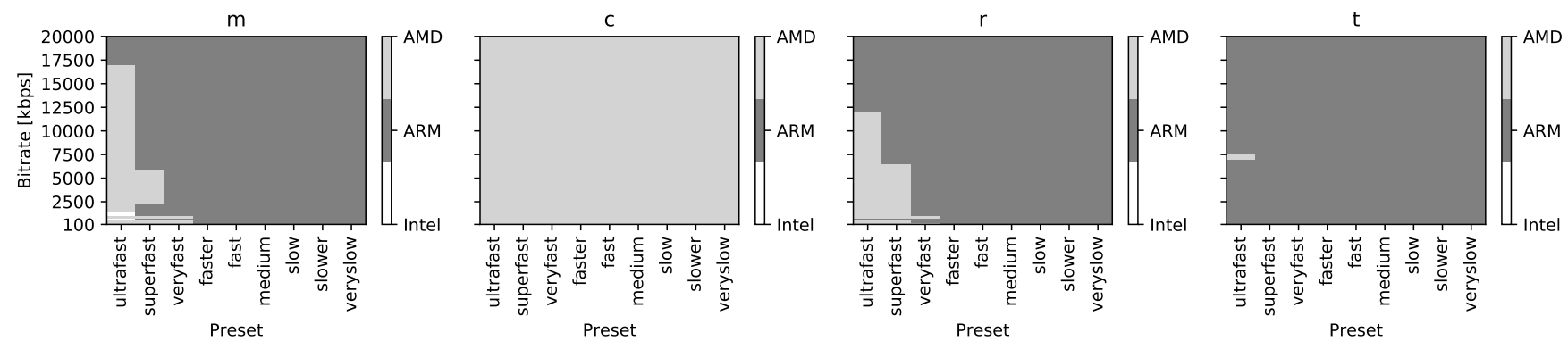

(b) Lowest encoding cost.

Figure 5: Reference table for fastest encoding times and lowest cost for four instance families with different presets, bitrates, and $\times 264$ codec.

b) $\times 265$ codec: We recommend $\times 86$ instead of Arm instances, as the $\times 265$ implementation in the latest FFmpeg version is not yet optimized for Arm. Among $\times 86$ instances, we recommend the AMD based c5a.2xlarge, which is on average $4.67 \%$ faster than the Intel based c5.2xlarge instance. Among the Arm instances, we recommend $\mathrm{m} 6 \mathrm{~g} .2 \times \mathrm{large}$ as it achieves the fastest encoding times in most experiments.

\section{2) Low cost encoding:}

a) $\times 264$ codec: Overall and as presented in Figure $5 \mathrm{~b}$. we recommend $\times 86$ instances for bitrates lower than $8 \mathrm{Mbps}$ and presets between "ultrafast" and "superfast". Among all $\times 86$ instances, we recommend to use $C$ instances and to prioritize the AMD based c5a instance. We recommend Arm instances for $m, r$, and $t$ instances especially for presets between "very fast" and "veryfast". In particular, Arm instances reduce the encoding cost of up to $33.63 \%$ compared to $\times 86$. We observe that $\mathrm{c} 5 \mathrm{a}$ achieves the lowest encoding cost among x86, and $4 \mathrm{~g} .2 \mathrm{xlarge}$ among Arm instances.

b) $\times 265$ codec: We recommend using $\times 86$ instances that generate on average $382.58 \%$ lower encoding costs than Arm instances. The c $5 \mathrm{a} .2 \mathrm{xl}$ arge instance achieves $15.65 \%$ lower average cost among $\times 86$ instances and on average $13.65 \%$ lower cost than $\mathrm{c} 5.2 \times$ large instances. Among Arm instances, the $t 4 \mathrm{~g} .2 \times$ large reveals lowest cost in all experiments, but the advantage is just $1.34 \%$.

\section{B. Discussion}

The pricing model of AWS ranks the instances in descending order with Intel $\times 86$ instances with highest cost, followed by AMD based $\times 86$ as middle cost, and Arm instances as low-cost alternatives. We observe that the instance performance primarily follows this pricing model. However, as cost and performance are two conflicting objectives, we also analysed the cost to performance ratio. We revealed that for specific settings and codecs the low cost Arm instances can outperform faster and higher price $\times 86$ instances. We explain this observation by the different instruction set architecture (ISA) of both processors types.

In particular, the $\times 86$ CPUs use Complex Instruction Set Computing (CISC) while Arm uses Reduced Instruction Set Computing (RISC). With other words, the former uses more complex instructions with several cycles while Arm uses only one cycle to execute a single instruction. Consequently, we identified the software support for Arm instances as an important aspect that affects the performance. For example, we observed a high performance discrepancy between Arm and $x 86$ instances for the compute intensive $x 265$ codec. We explain the discrepancy through the fact that the FFmpeg 4.3 is not yet optimised for Arm instances.

Besides, the performance depends on the hardware allocation of the cloud provider. For example, the default allocation of Amazon EC2 uses hyper-threading that assigns on $\times 86$ instances one logical hyper-threaded core to a vCPU (except t2). In contrast, the Arm instances assign one physical core 
Table III: Encoding recommendation summary.

\begin{tabular}{|c|cc|c|}
\hline Codec Goal & \multicolumn{2}{|c|}{ Fast encoding } & \multicolumn{2}{c|}{ Low cost } \\
\hline$x 264$ & $\begin{array}{l}\text { x86 } \\
\text { Arm }\end{array}(\mathrm{c} 5 \mathrm{a})$ & Arm ( $6 \mathrm{~g})$
\end{tabular}

to a vCPU [7] in the absence of hyper-threading. This fact enables companies ${ }^{1}$, such as Snap Inc reducing CPU utilization by roughly $10 \%$, Honeycomb.io running $30 \%$ fewer instances, and NextRoll saving up to $50 \%$ cost compared to previous generation EC2 instances for their workloads.

Overall, with continuously improving software support and optimisation for Arm, we forecast a decreasing performance difference between Arm and $\times 86$ instances which also applies for video encoding tasks.

\section{CONCLUSION}

In this paper, we provide a performance analysis for video encoding tasks of Arm and $x 86$ instances of four Amazon EC2 instance families with three different processors. We conducted a total of 20.520 experiments in two evaluation scenarios for the two most widely used video codecs.

Table III summarises our recommendations based on the evaluation results related to the encoding time and cost.

The evaluation results clearly reveal that the Arm instances can achieve faster encoding times at a lower cost than the corresponding $\times 86$ instances. However, the encoding performance of Arm and $\times 86$ instances depends on many factors such as bitrate, preset, and codec. Independently from the codec, we show that the $x 86 \mathrm{C}$ instance and specifically the AMD based $\mathrm{c} 5 \mathrm{a}$ instance achieves fast encoding times at a lower cost in most experiments, which makes it suitable for general encoding use.

In summary, regarding the measured encoding performance potential for specific encoding settings and the continuously improving support of Arm instances, we forecast a decreasing performance difference between Arm and $\times 86$ instances.

In the future, we plan to extend our analysis with different emerging codec implementations and longer video segments to generalize our recommendations. Furthermore, we will perform an in-depth analysis of overprovisioning by evaluating x86 instances with various CPU options [7] for the fastest instance setup.

\section{REFERENCES}

[1] Use Spot Instance Pricing for Your Video Encoding Workflows with Bitmovin. https://aws.amazon.com/ru/blogs/startups/use-spotinstance-pricing-for-your-video-encoding-workflows-with-bitmovincontainerized-encoding 2017. [Online; accessed 08-September-2020].

[2] Arm's market share and targets across key technology markets in 2018 and 2028 fiscal years. https://www.statista.com/statistics/1132112/armmarket-share-targets/ 2020. [Online; accessed 29-September-2020].

[3] AWS Graviton Processor. Enabling the best price performance in Amazon EC2. https://aws.amazon.com/ec2/graviton 2020. [Online; accessed 08-September-2020].

1 https://aws.amazon.com/ec2/graviton
[4] Cisco annual internet report (2018-2023). Technical report, Cisco, 170 West Tasman Dr. San Jose, CA 95134 USA, 2020.

[5] FFmpeg Codecs Documentation. https://ffmpeg.org/ffmpegcodecs.html 2020. [Online; accessed 14-September-2020].

[6] H.264 Video Encoding Guide. https://trac.ffmpeg.org/wiki/Encode/ H.264 2020. [Online; accessed 10-September-2020].

[7] Optimizing CPU options. https://docs.aws.amazon.com/AWSEC2/latest/ UserGuide/instance-optimize-cpu.html 2020. [Online; accessed 4October-2020].

[8] x265 documentation. Release Notes. https://x265.readthedocs.io/en/ default/releasenotes.html 2020. [Online; accessed 14-September-2020].

[9] B. Bross, J. Chen, and S. Liu. Versatile Video Coding (Draft 7). Document, JVET-P2001, Geneva, CH, October 2019.

[10] Abdelhak Bentaleb, Bayan Taani, Ali C. Begen, Christian Timmerer, and Roger Zimmermann. A survey on bitrate adaptation schemes for streaming media over HTTP. IEEE Commun. Surv. Tutorials, 21(1):562_ $585,2019$.

[11] Nassima Bouzakaria, Cyril Concolato, and Jean Le Feuvre. Overhead and performance of low latency live streaming using MPEG-DASH. In 5th International Conference on Information, Intelligence, Systems and Applications, IISA 2014, Chania, Crete, Greece, July 7-9, 2014, pages 92-97, 2014.

[12] Yue Chen, Debargha Mukherjee, Jingning Han, Adrian Grange, Yaowu $\mathrm{Xu}$, Zoe Liu, Sarah Parker, Cheng Chen, Hui Su, Urvang Joshi, ChingHan Chiang, Yunqing Wang, Paul Wilkins, Jim Bankoski, Luc N. Trudeau, Nathan E. Egge, Jean-Marc Valin, Thomas Davies, Steinar Midtskogen, Andrey Norkin, and Peter De Rivaz. An overview of core coding tools in the AV1 video codec. In 2018 Picture Coding Symposium, PCS 2018, San Francisco, CA, USA, June 24-27, 2018, pages $41-45,2018$.

[13] Michael Ferdman, Almutaz Adileh, Yusuf Onur Koçberber, Stavros Volos, Mohammad Alisafaee, Djordje Jevdjic, Cansu Kaynak, Adrian Daniel Popescu, Anastasia Ailamaki, and Babak Falsafi. Clearing the clouds: a study of emerging scale-out workloads on modern hardware. In Proceedings of the 17th International Conference on Architectural Support for Programming Languages and Operating Systems, ASPLOS 2012, London, UK, March 3-7, 2012, pages 37-48, 2012.

[14] Q. Huangyuan, L. Song, Z. Luo, X. Wang, and Y. Zhao. Performance evaluation of H.265/MPEG-HEVC encoders for $4 \mathrm{~K}$ video sequences. In Signal and Information Processing Association Annual Summit and Conference (APSIPA), 2014 Asia-Pacific, pages 1-8, 2014.

[15] ITU-T. Subjective video quality assessment methods for multimedia applications. Recommendation ITU-T P.910, International Telecommunication Union, Geneva, April 2008.

[16] ITU-T. H.264 : Advanced video coding for generic audiovisual services. Recommendation T-REC-H.264-201906-I, International Telecommunication Union, Geneva, April 2019.

[17] Qingye Jiang, Young Choon Lee, and Albert Y. Zomaya. Scalable video transcoding in public clouds. In 19th IEEE/ACM International Symposium on Cluster, Cloud and Grid Computing, CCGRID 2019, Larnaca, Cyprus, May 14-17, 2019, pages 70-75, 2019.

[18] Qingye Jiang, Young Choon Lee, and Albert Y. Zomaya. The power of ARM64 in public clouds. In 20th IEEE/ACM International Symposium on Cluster, Cloud and Internet Computing, CCGRID 2020, Melbourne, Australia, May 11-14, 2020, pages 459-468, 2020.

[19] Dragi Kimovski, Roland Mathá, Josef Hammer, Narges Mehran, Hermann Hellwagner, and Radu Prodan. Cloud, fog or edge: Where to compute? IEEE Internet Computing, 2021.

[20] Maria G. Koziri, Panos K. Papadopoulos, Nikos Tziritas, Thanasis Loukopoulos, Samee U. Khan, and Albert Y. Zomaya. Efficient cloud provisioning for video transcoding: Review, open challenges and future opportunities. IEEE Internet Comput., 22(5):46-55, 2018.

[21] Pierre Lebreton, Werner Robitza, and Steve Göring. Command-line tool for calculating SI and TI according to ITU-T P.910. https://github.com/ Telecommunication-Telemedia-Assessment/SITI] 2019. [Online; accessed 25-July-2020].

[22] Stefan Lederer, Christopher Müller, and Christian Timmerer. Dynamic adaptive streaming over HTTP dataset. In Proceedings of the Third Annual ACM SIGMM Conference on Multimedia Systems, MMSys 2012, Chapel Hill, NC, USA, February 22-24, 2012, pages 89-94, 2012.

[23] Xiangbo Li, Mohsen Amini Salehi, Magdy A. Bayoumi, Nian-Feng Tzeng, and Rajkumar Buyya. Cost-efficient and robust on-demand video transcoding using heterogeneous cloud services. IEEE Trans. Parallel Distrib. Syst., 29(3):556-571, 2018. 
[24] Xiangbo Li, Mohsen Amini Salehi, Yamini Joshi, Mahmoud K. Darwich, Brad Landreneau, and Magdy A. Bayoumi. Performance analysis and modeling of video transcoding using heterogeneous cloud services. IEEE Trans. Parallel Distrib. Syst., 30(4):910-922, 2019.

[25] Andrea Lottarini, Alex Ramírez, Joel Coburn, Martha A. Kim, Parthasarathy Ranganathan, Daniel Stodolsky, and Mark Wachsler. vbench: Benchmarking video transcoding in the cloud. In Proceedings of the Twenty-Third International Conference on Architectural Support for Programming Languages and Operating Systems, ASPLOS 2018, Williamsburg, VA, USA, March 24-28, 2018, pages 797-809, 2018.

[26] Ikuo Magaki, Moein Khazraee, Luis Vega Gutierrez, and Michael Bedford Taylor. ASIC clouds: Specializing the datacenter. In 43rd ACM/IEEE Annual International Symposium on Computer Architecture, ISCA 2016, Seoul, South Korea, June 18-22, 2016, pages 178-190, 2016.

[27] Cise Midoglu, Anatoliy Zabrovskiy, Ozgu Alay, Daniel Hoelbling-Inzko, Carsten Griwodz, and Christian Timmerer. Docker-based evaluation framework for video streaming qoe in broadband networks. In Proceedings of the 27th ACM International Conference on Multimedia, MM 2019, Nice, France, October 21-25, 2019, pages 2288-2291, 2019.

[28] Debargha Mukherjee, Jingning Han, Jim Bankoski, Ronald Bultje, Adrian Grange, John Koleszar, Paul Wilkins, and Yaowu Xu. A technical overview of vp9-the latest open-source video codec. SMPTE Motion Imaging Journal, 124(1):44-54, 2015.

[29] Dieison Silveira, Marcelo Schiavon Porto, and Sergio Bampi. Performance and energy consumption analysis of the X265 video encoder. In 25th European Signal Processing Conference, EUSIPCO 2017, Kos, Greece, August 28 - September 2, 2017, pages 1519-1523, 2017.

[30] Iraj Sodagar. The MPEG-DASH standard for multimedia streaming over the internet. IEEE MultiMedia, 18(4):62-67, 2011.

[31] Gary J. Sullivan, Jens-Rainer Ohm, Woojin Han, and Thomas Wiegand.
Overview of the high efficiency video coding (HEVC) standard. IEEE Trans. Circuits Syst. Video Technol., 22(12):1649-1668, 2012.

[32] Jan Ten Sythoff. The Total Economic Impact Of Arm Neoverse. Technical report, Study Commissioned By Arm, June 2020.

[33] Christian Timmerer, Daniel Weinberger, Martin Smole, Reinhard Grandl, Christopher Müller, and Stefan Lederer. Live transcoding and streamingas-a-service with MPEG-DASH. In 2015 IEEE International Conference on Multimedia \& Expo Workshops, ICME Workshops 2015, Turin, Italy, June 29 - July 3, 2015, pages 1-4, 2015.

[34] Dmitriy Vatolin, Dmitriy Kulikov, Egor Sklyarov, Sergey Zvezdakov, and Anastasia Antsiferova. Video Transcoding Clouds Comparison 2019. Technical report, Moscow State University, 112019.

[35] Thomas Wiegand, Gary J. Sullivan, Gisle Bjøntegaard, and Ajay Luthra. Overview of the H.264/AVC video coding standard. IEEE Trans. Circuits Syst. Video Technol., 13(7):560-576, 2003.

[36] Anatoliy Zabrovskiy, Christian Feldmann, and Christian Timmerer. Multi-codec DASH dataset. In Proceedings of the 9th ACM Multimedia Systems Conference, MMSys 2018, Amsterdam, The Netherlands, June 12-15, 2018, pages 438-443, 2018.

[37] Anatoliy Zabrovskiy, Christian Feldmann, and Christian Timmerer. A practical evaluation of video codecs for large-scale HTTP adaptive streaming services. In 2018 IEEE International Conference on Image Processing, ICIP 2018, Athens, Greece, October 7-10, 2018, pages 9981002, 2018.

[38] Anatoliy Zabrovskiy, Evgeny Kuzmin, Evgeny Petrov, Christian Timmerer, and Christopher Müller. Advise: Adaptive video streaming evaluation framework for the automated testing of media players. In Proceedings of the 8th ACM on Multimedia Systems Conference, MMSys 2017, Taipei, Taiwan, June 20-23, 2017, pages 217-220, 2017. 